\title{
Ewing's sarcoma of the spinal epidural space: report of two cases
}

\author{
BER D W. SCHEITHAUER A ND BARBARA M. EGBERT \\ From the Department of Pathology (Neuropathology), Stanford University School of Medicine, \\ Stanford, California, and the Department of Pathology, Veterans Administration Hospital, \\ Palo Alto, California, USA
}

S UMMARY Two new cases of primary extraosseous Ewing's sarcoma of the spinal epidural space, and their histogenesis and differential diagnosis are described. The diagnosis of Ewing's sarcoma, which is essentially an undifferentiated tumour, depends largely on the exclusion of several other neoplasms with morphological similarities. With these two cases, 43 extraosseous Ewing's sarcomas have been reported to date, seven of which were epidural in location.

Most patients with Ewing's sarcoma present with the clinical and radiographic features of a primary osseous tumour. However, Tefft et al. (1969), in a discussion of round cell tumours of the paravertebral region in children, described two patients with extraosseous paravertebral and contiguous epidural neoplasms with the morphological features of Ewing's sarcoma. Angervall and Enzinger (1975) subsequently established the entity of extraosseous Ewing's sarcoma by describing 39 extraskeletal examples. These occurred most frequently in young adults. Thirteen involved the paraspinal region, and of these, three were epidural. We report an additional two patients with primary extraosseous Ewing's sarcoma involving the spinal epidural space.

\section{Case reports}

CASE 1

Two months before referral to the Stanford University Medical Center, the patient, an 18 year old Caucasian male student, complained of radiating pain along the left iliac crest and anterior aspect of the thigh. This was followed one month later by low back pain which failed to respond to symptomatic treatment. Physical examination on admission disclosed focal tenderness to palpation over the L4-5 vertebral region. Although the patient had complained of numbness and tingling

Address for reprint requests: Dr Bernd W. Scheithauer, Department of Pathology (Neuropathology), Stanford University Medical Center, Stanford, California 94305, USA.

Accepted 23 June 1978 of the lateral aspect of the thigh, neurological testing showed neither motor nor sensory loss. There was no bowel or bladder dysfunction. Low back pain was worsened on straight leg raising. Radiography showed no osseous lesion, but demonstrated complete myelographic block at the L1 vertebral level. Laminectomy disclosed a soft vascular circumscribed grey-tan tumour, $20 \mathrm{~mm}$ in width, compressing the left anterolateral surface of the dura mater at the L1 vertebral level. The tumour was completely resected; there was no visible bone involvement. Extensive postoperative radiographic studies showed no other site of tumour. The patient received opposed anteriorposterior field radiation therapy to the thoracolumbar spine- 5400 rads were administered between the vertebral levels T12 to L2. Thereafter, pulse chemotherapy with vincristine, actinomycin$D$, and cytoxan was given. The patient is free from disease without symptoms 16 months after the initial diagnosis.

\section{CASE 2}

The patient, a 27 year old woman, first noted incoordination and increasing bilateral leg weakness in the spring of 1967. One year later, increasingly severe back pain, accompanied by bilateral leg paraesthesiae, prompted radiographic studies which disclosed a paravertebral mass at the T6 vertebral level, associated with a complete extradural block at the vertebral levels T4-6. There was no radiographic evidence of bone involvement. At laminectomy, a paraspinal and partially epidural dumb-bell shaped mass was found; neither com- 
ponent was excised totally. No vertebral or rib involvement was found. The patient received local radiation therapy (5000 rads), at the conclusion of which chest radiographs showed complete resolution of the paravertebral mass. The patient remained asymptomatic until June 1970, when she developed increasingly severe back pain. Chest radiography in September 1970 showed a right upper lobe mass and a mass in the right fifth rib, which was interpreted as a metastasis. Additional local radiation therapy and chemotherapy (vincristine, actinomycin-D, and cytoxan) were administered. In December 1970 there was no radiographic evidence of tumour and the patient was asymptomatic. Chemotherapy was discontinued. The patient remained well during the next five years until December 1975, when radiographs disclosed a lytic vertebral lesion. A thoracotomy disclosed tumour involving the vertebra and chest wall. A liver scan showed a pattern of diffuse parenchymal disease. The patient underwent 12 monthly cycles of chemotherapy with the same agents as before. Radiographic follow-up in January 1978 showed near total resolution of the lesions.

\section{PATHOLOGY}

Both tumours consisted of a uniform population of small round cells with pale indistinct cytoplasm. The cells, somewhat larger than lymphocytes, contained round to ovoid nuclei with distinct nuclear membranes, delicate, evenly dispersed chromatin, and small nucleoli. Mitotic figures were numerous. The vesicular cytoplasm contained large quantities of PAS-positive, diastase-labile glycogen. Toluidine blue Nissl stained sections showed no abnormality, and Bielschowsky silver impregnation preparations showed no neurofibrils. The cells were disposed both in sheets (Fig. 1) and in irregular clusters, separated by fibrovascular septa. Although small numbers of tumour cells were enmeshed in a perivascular reticulin network that extended for a short distance from these septa, no reticulin fibres were present within the cellular sheets and lobules (Fig. 2). Some tumour cells tended to arrange themselves around capillary blood vessels, but others showed a distinct tendency to rosette formation. This pattern was readily seen in numerous sections and was caused by the circumferential orientation of cells around minute foci of degenerating tumour cells (Fig. 3). Small numbers of cells with angular or elongate pyknotic nuclei and sparse eosinophilic cytoplasm were also seen. These apparently degenerating cells bore no consistent relationship to vessels (Fig. 4). Scattered macrophages with abundant

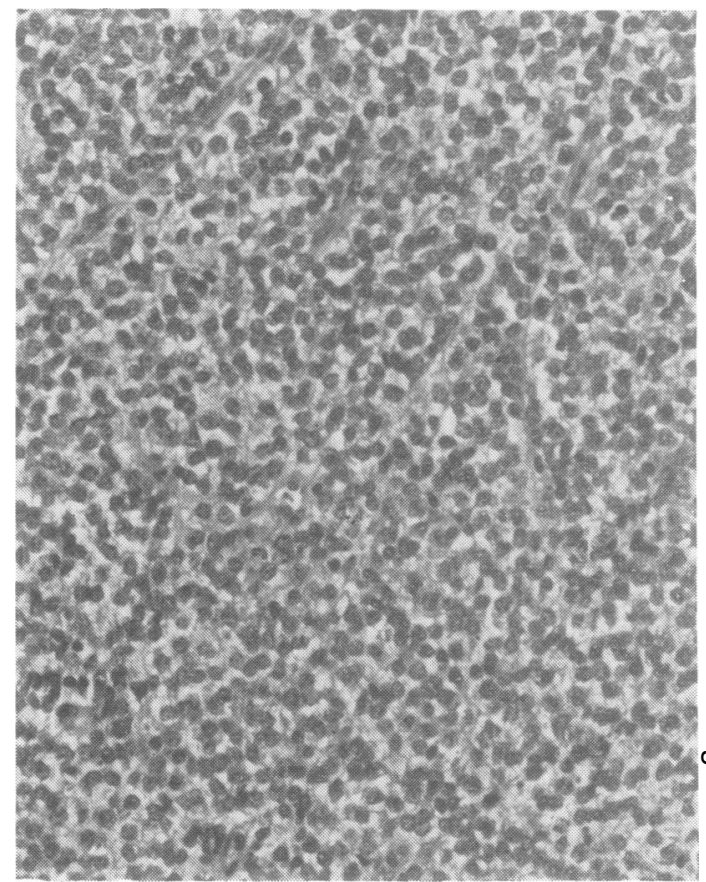

Fig. 1 Case 1. Uniform population of small cells disposed in sheets. Haematoxylin-Van Gieson $\times 190$.

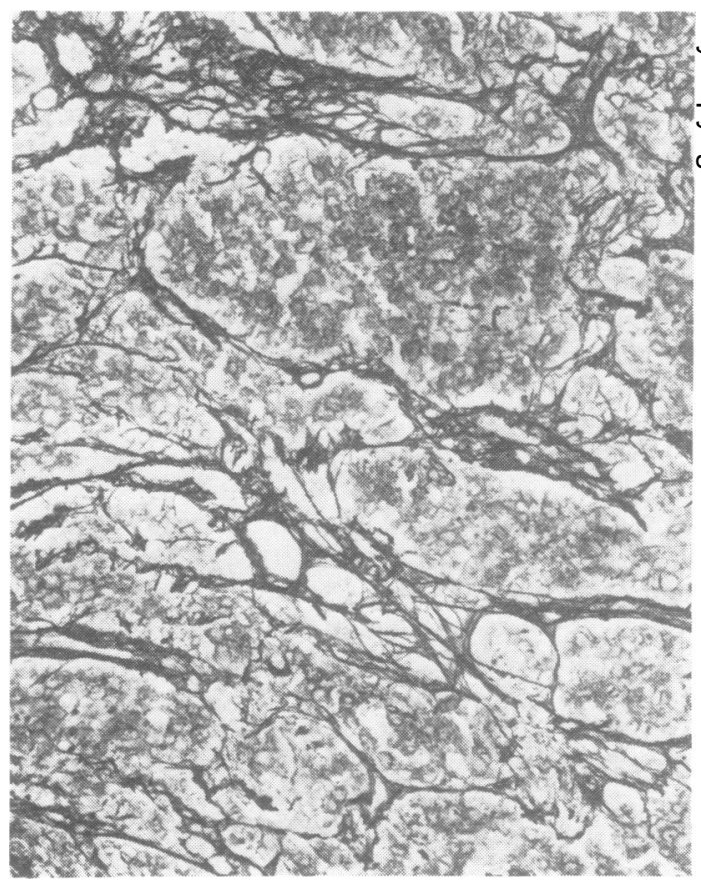

Fig. 2 Case 1. Lobular arrangement of tumour cells with lack of intercellular reticulin. Gordon-Sweets' silver method for reticulin $\times 190$. 


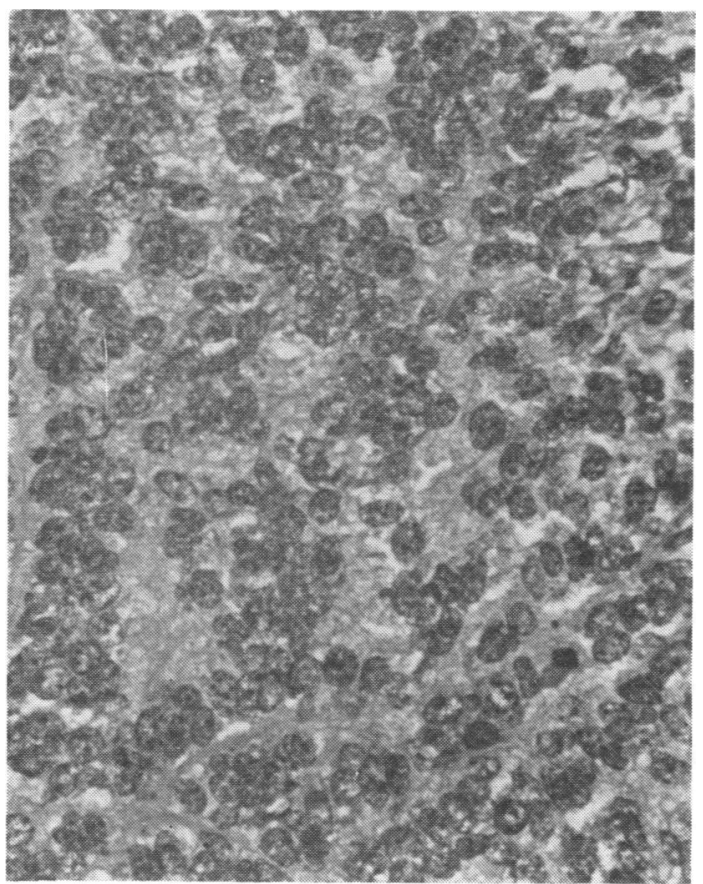

Fig. 3 Case 2. Arrangement of tumour cells around foci of degenerating cells to form "granular rosettes." HVG 380 .

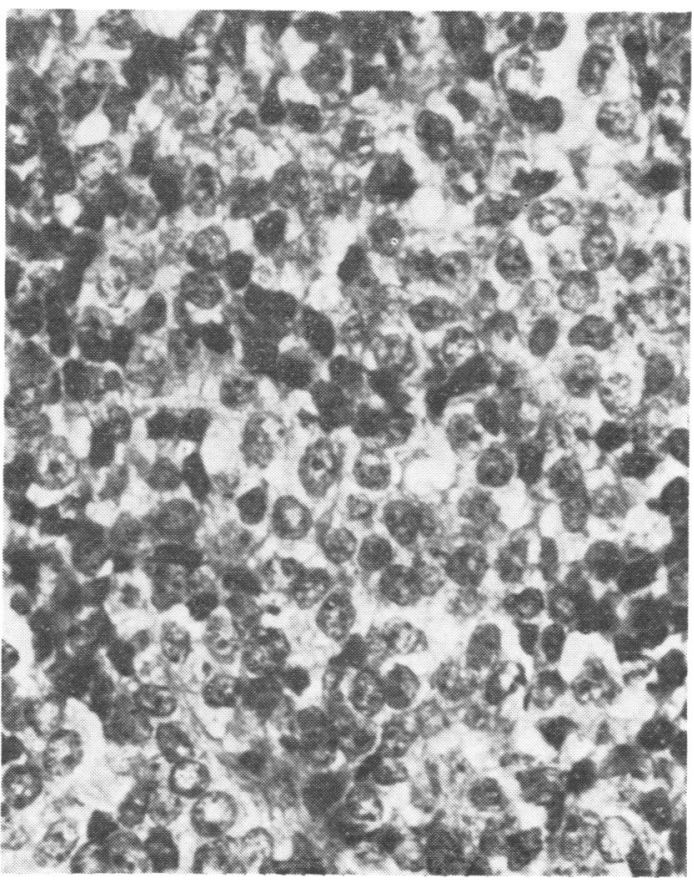

Fig. 4 Case 2. Admixture of typical light cells and small pyknotic cells. $H V G \times 480$. cytoplasm were identified among the tumour cells. No production or invasion of bone or cartilage was seen. Necrosis was patchy. No calcification was found.

\section{Discussion}

Hamby (1935, 1944), in an exhaustive literature review of intraspinal tumours of childhood, summarised 214 cases, of which $88(41 \%)$ involved the epidural space. The relative frequency of tumour types in this location was as follows: sarcomas (39), schwannomas (11), chloromas (8), dermoid cysts (6), sympathetic tumours (5), vascular tumours (4), fibrous tumours (3), meningiomas (2), and lipomas (2). Four $(10 \%)$ of the epidural sarcomas demonstrated a contiguous paravertebral component. By contrast, in the adult population, metastatic carcinoma from the lung or breast, lymphoma, and myeloma are the most frequent neoplasms involving the spinal epidural space. In all series, most tumours affect the thoracic region, the lumbar level being the next most frequent. No consistent relationship exists between tumour type, primary site, and the level of cord compression.

Ewing's sarcoma comprises $5 \%$ of the skeletal tumours, occurs most frequently in males in the first to third decades, and, although it has been described in nearly all the bones of the body, arises preferentially in the medullary cavity of the diaphysis of the tubular bones (Dahlin et al., 1961). Apart from absence of male sex predominance and from its occurrence in a slightly older age population (average 20 years), the biological behaviour and prognosis of extraosseous Ewing's sarcoma are very similar to those of the skeletal examples. Tumours in both locations follow a rapid course with metastasis to lung and bone. Occasionally, Ewing's sarcoma may arise in the vertebral body, and encroach secondarily upon the epidural space (Anderson and Carson, 1953; Rand and Rand, 1960 ), or sometimes the tumour may be situated largely in the periosteum and bone cortex. Therefore, it is essential to rule out vertebral involvement before making the diagnosis of extraosseous Ewing's sarcoma.

The histogenesis of Ewing's sarcoma has long been a subject of debate. Whereas Ewing (1924), who noted the presence of tumour cells in vascular spaces, believed that the tumour arose from endothelial cells, subsequent authors have suggested an origin from mesenchymal stem cells (Melnick, 1922; Oberling and Raileanu, 1932), reticulum cells (Friedman and Gold, 1968; Takayama and Sugawa, 1970) and primitive myeloid cells (Roome and Delaney, 1932; Kadin and Bensch, 1971; Hou- 
Jensen et al., 1972). Kadin and Bensch (1971), in a combined electron microscopic and tissue culture study of a typical Ewing's sarcoma, reported the acquisition by tumour cells of hydrolytic enzymes, as well as azurophylic granules. They suggested that Ewing's tumour is a neoplasm of myelogenous origin. Division of opinion also exists with regard to the nature of the dark cells in Ewing's sarcoma and their possible relationship to the more numerous light cells. On the basis of their ultrastructural features, Friedman and Gold (1968) regard the dark cells as more differentiated forms, with features of reticulum cells, which arise through transitional forms from the light cells, which they considered mesenchymal stem cells. A similar view is expressed by Nakayama et al. (1975). Kadin and Bensch (1971) regard the dark cells as degenerating rather than as differentiated cells.

The diagnosis of Ewing's sarcoma, which is essentially an undifferentiated tumour, depends in large measure on the exclusion of several other neoplasms with morphological similarities-that is, reticulum cell sarcoma (histiocytic lymphoma), neuroblastoma, alveolar rhabdomyosarcoma, and undifferentiated carcinoma. Abundant intracytoplasmic glycogen, the characteristic morphological feature of Ewing's sarcoma, is uncommon in neoplasms in general, and its demonstration is crucial in the differential diagnosis. The clinical importance and histological method of distinguishing reticulum cell sarcoma of bone from Ewing's sarcoma are discussed by Schajowicz (1959). Reticulum cell sarcoma (histiocytic lymphoma) lacks the lobular architecture of Ewing's sarcoma, has a variable quantity of intercellular reticulin, and is composed of larger cells with vesicular nuclei and prominent nucleoli. PAS stains demonstrate little or no cytoplasmic glycogen, whereas the methyl green-pyronine stain for ribonuclear protein is positive. Although patients with neuroblastoma are frequently younger than those with extraosseous Ewing's sarcoma and show involvement of the adrenal glands or sympathetic ganglia, both lesions may present with epidural and contiguous paraspinal tumour involvement (Ingraham and Matson, 1954), and may show rosettes on microscopy. Rosette formation in Ewing's sarcoma has been described previously (Foote and Anderson, 1941; Gharpure, 1941). The rosettes of Ewing's sarcoma superficially resemble those of neuroblastoma, which in routine sections stained with haematoxylin eosin show no sharp luminal cell boundary, but rather the convergence of interlacing cell processes. The rosettes in our two cases of Ewing's sarcoma were less well formed than those of neuroblastoma, and were composed of tumour cells arranged about foci of granular debris derived from degenerating tumour cells. Bielschowsky silver impregnations, particularly when performed on frozen tissue, either fresh or fixed, should help in differentiating these "granular rosettes" from the fibrillar rosettes of Homer Wright.

Comparative ultrastructural studies of Ewing's sarcoma and neuroblastoma were performed by Friedman and Hanaoka (1971), and more recently by Nakayama et al. (1975). The cells of Ewing's sarcoma were shown to contain abundant aggregated intracytoplasmic glycogen particles, whereas neuroblastoma cells demonstrated scattered dense core granules as well as processes containing neurofilaments and microtubules. The importance of electron microscopy in the distinction of these two tumours is underlined by the recent report of a glycogen-containing neuroblastoma with clinical and histopathological features of Ewing's sarcoma (Triche and Ross, 1978). The distinction of Ewing's sarcoma from alveolar rhabdomyosarcoma is lesso of a problem in that the latter is composed of lesso uniform, darkly staining cells, possessing eosinophilic cytoplasm containing moderate quantities of stored glycogen. Both cytoplasmic cross striationso and the distinct alveolar pattern with loss of central cohesion are not seen in Ewing's sarcoma. The exclusion of undifferentiated carcinoma be $z$ comes a consideration in older patients and mayo require electron microscopy. The finding of welfo formed desmosomes with tonofilaments, characteristic of epithelial cells, confirms the diagnosis. The demonstration of specific granules and enzyme histochemical reactions peculiar to acute leukaemic infiltrates should facilitate their distinction from Ewing's sarcoma.

We have presented two further cases of primary extraosseous Ewing's sarcoma, both involving the epidural space. Although the follow-up is rather short, the disease-free course of case 1 is exceptional and is likely to reflect the completeness of surgical excision as well as the efficacy of aggressive irradiation and sequential adjuvant chemotherapy as recommended by Rosen et al. (1978). The late recurrence and prolonged course of the disease in case 2 is also unusual, but the development of pulmonary and osseous metastases is characteristic of Ewing's sarcoma.

With the inclusion of these cases, 43 extraosseous Ewing's sarcomas have been reported to date (Tefft et al., 1969; Angerval and Enzinger, 1975); seven $(17 \%)$ were epidural in location, three of these were contiguous with, and may represent extension from, tumour in the paravertebral area. Six of the seven cases involved the thoracolumbar region. 
This study was supported in part by Graduate Neuropathology Training Grant 5 TO1 NS-0550011 of the National Institute of Neurological and Communicative Diseases and Stroke, USPHS. We would like to thank Dr L. S. Hurwitz and Dr W. V. Dolan, Milwaukee, Wisconsin for making the material of case 2 available to us, and $\operatorname{Dr} \mathrm{L}$. J. Rubinstein and Dr H. Urich for critical review of the manuscript.

\section{References}

Anderson, F. M., and Carson, M. J. (1953). Spinal cord tumors in children: review of the subject and presentation of twenty-one cases. Journal of Pediatrics, 43, 190-207.

Angervall, L., and Enzinger, F. N. (1975). Extraskeletal neoplasm resembling Ewing's sarcoma. Cancer, 36, 240-251.

Dahlin, D. C., Coventry, N. B., and Scanlon, P. W. (1961). Ewing's sarcoma. A critical analysis of 165 cases. Journal of Bone and Joint Surgery, 43-A, 185192.

Ewing, J. (1924). Further report on endothelial myeloma of bone. Proceedings of the New York Pathological Society, 23, 93-101.

Foote, F. W., and Anderson, H. R. (1941). Histogenesis of Ewing's tumor. American Journal of Pathology, 17, 497-509.

Friedman, B., and Gold, H. (1968). Ultrastructure of Ewing's sarcoma of bone. Cancer, 22, 307-322.

Friedman, B., and Hanaoka, H. (1971). Round-cell sarcoma of bone. Journal of Bone and Joint Surgery, 53A, 1118-1136.

Gharpure, V. V. (1941). Endothelial myeloma (Ewing's tumor of bone). American Journal of Pathology, 17, 503-507.

Hamby, W. B. (1935). Tumors of the spinal canal in childhood. An analysis of the literature and report of a case. Journal of Nervous and Mental Disease, 81, 24-42.

Hamby, W. B. (1944). Tumors of the spinal canal in childhood. Analysis of the literature of subsequent decade (1933-1942). Report of a case of meningitis due to an intramedullary epidermoid communicating with a dermal sinus. Journal of Neuropathology and Experimental Neurology, 3, 397-412.
Hou-Jensen, K., Priori, E., and Dmochowski, L. (1972). Studies on ultrastructure of Ewing's sarcoma of bone. Cancer, 29, 280-286.

Ingraham, F. D., and Matson, D. D. (1954). Neurosurgery of Infancy and Childhood. Charles C. Thomas: Springfield, Illinois.

Kadin, N., and Bensch, K. G. (1971). On the origin of Ewing's tumor. Cancer, 27, 257-273.

Melnick, P. J. (1922). Histogenesis of Ewing's sarcoma of bone with postmortem report of a case. American Journal of Cancer, 19, 553-563.

Nakayama, I., Studa, N., Nuta, H., Fujii, H., Tsuji, K., Matsuo, T., and Takahara, O. (1975). Fine structural comparison of Ewing's sarcoma with neuroblastoma. Acta Pathologica Japonica, 25, 251-268.

Oberling, C., and Raileanu, C. (1932). Nouvelles recherches sur les reticulosarcomes de la moelle osseuse (sarcomes d'Ewing). Bulletin de l'Association Française pour l'Etude du Cancer, 21, 333347.

Rand, R. W., and Rand, C. W. (1960). Intraspinal Tumors of Childhood. Charles C. Thomas: Springfield, Illinois.

Roome, N. W., and Delaney, P. A. (1932). Undifferentiated round cell sarcoma of ilium (Ewing's tumour) containing hemopoetic elements. American Journal of Cancer, 16, 386-398.

Rosen, G., Caparros, B., Mosende, C., McCormick, B., Huvos, A. G., and Marcove, R. C. (1978). Curability of Ewing's sarcoma and considerations for future therapeutic trials. Cancer, 41, 888-889.

Schajowicz, F. (1959). Ewing's sarcoma and reticulum-cell sarcoma of bone with special reference to the histochemical demonstration of glycogen as an aid to differential diagnosis. Journal of Bone and Joint Surgery, 41A, 349-356.

Takayama, S., and Sugawa, I. (1970). Electron microscopic observation of Ewing's sarcoma-a case report. Acta Pathologica Japonica, 21, 87-101.

Tefft, M., Vawter, G. F., and Mitus, A. (1969). Paravertebral "round cell" tumors in children. Radiology, 92, 1501-1509.

Triche, T. J., and Ross, W. E. (1978). Glycogencontaining neuroblastoma with clinical and histopathologic features of Ewing's sarcoma. Cancer, 41, 1425-1432. 